Jordan B. L. Smith, Elaine Chew, \& Gérard Assayag (editors), Mathemusical conversations: Mathematics and computation in music performance and composition. Singapore: World Scientific Publishing, 2016. ISBN 978-981-3140-09-7 (hardcover) GBP 106.00. ISBN 978-9813140-11-0 (ebook) GBP 85.00. https://doi.org/10.1142/10046

Mathemusical Conversations is a collection of papers and essays based on a workshop that took place on 1315 February 2015 at the Yong Siew Toh Conservatory of Music, part of the National University of Singapore. The aim of this workshop was to "celebrate the understanding of the essence of music through mathematics, with a special focus on performance and composition" ( $\mathrm{p}$. ix). The resulting book comprises two forewords plus fifteen chapters, organised into five parts: I. Mathemusical Engagement; II. Mathemusical Creativity; III. Shaping Performance; IV. Educating the Mathemusical; V. Geometries. These chapters cover a broad array of current research areas in the overlap between music and mathematics.

\title{
Summary of contributions
}

\section{MATHEMUSICAL ENGAGEMENT}

Mathemusical Engagement comprises contributions from Paul Schoenfield, Eran Egozy, and Elaine Chew. Schoenfield discusses the relationship between beauty in mathematics and beauty in music. He takes a negative stance towards using mathematics as a method of composition, writing that it mostly results in "bad music and worse mathematics" (p. 6). However, he suggests that musical quality is intrinsically connected to patterns of expectation and surprisal that might be characterized in probability theory, after Meyer (1956, 1967) [1]. Schoenfield then discusses what makes a mathematical theorem beautiful, suggesting that both simplicity and the element of surprise are important contributors. He makes an interesting comparison between the process of finding a new proof for a previously proved mathematical theorem (reproving) and composing a new piece in a historical musical style (pastiche composition). Reproving is a well-respected mathematical activity, whereas pastiche composition is traditionally seen as having low artistic value; however, Schoenfield suggests that we are now entering an age of musical reproving, where composers revisit historical musical styles.

Egozy writes about his experience as founder of Harmonix Music Systems, creating products designed to increase the accessibility of music-making for non-musicians. The chapter centers on five such products: The Axe, Amplitude, Karaoke Revolution, Guitar Hero/Rock Band, and Fantasia: Music Evolved. Through several case studies, Egozy illustrates the pervasive difficulty in balancing engagement with expressivity. Engaging games tend to require mechanics for objectively evaluating the player's actions against some pre-defined criteria. Since it is difficult to evaluate creativity objectively, games instead tend to evaluate players on lower-level aspects of performance, such as the extent to which timing or tuning deviates from that of a predefined "standard" performance. However, expressive musical performance typically requires the freedom to deviate from such standards. As a result, engaging music games tend to penalize creativity in the performer. Egozy presents Fantasia: Music Evolved as a partial solution to this problem: in this game, objectively scored performance regularly alternates with free performance, allowing the user to experience a mixture of engaging gameplay and expressive opportunities.

Chew writes about how mathematics can be used to create useful analogies for explaining musical structure, using two case studies from her previous work. The first is her Spiral Array Model (Chew, 2002), which combines the physical concept of the center of gravity (the average location of an object's mass) with a geometric model of tonal space, producing a model that can be used to visualize tonal trajectories throughout pieces of music. The second analogy is between the temporal progression of a musical performance and motion through a physical landscape. Chew recounts a previous study on tipping points (Chew, 2016), points of temporary stasis where a small perturbation would initiate a return of motion. In the physical world, a tipping point might be experienced by a train at the top of a rollercoaster; in the musical world, a tipping point might be experienced in the conclusion of a concerto cadenza, anticipating the return of the tonic. Chew gives a couple of musical examples of tipping points, and links these tipping points to tonal progressions as visualized by the Spiral Array Model. 


\section{MATHEMUSICAL CREATIVITY}

Mathemusical Creativity includes contributions from Gérard Assayag, Margaret Boden, and Moreno Andreatta. Assayag's chapter is based on a keynote presentation from the 2014 ICMC/SMC conference (Assayag, 2014), and concerns creative symbolic interaction, where a performer interacts with an artificial musical agent that exhibits autonomous musical development while remaining stylistically consistent with the performer. Assayag describes how he and his research group have attempted to achieve this goal through the creation of OMax, an interactive machine improvisation environment. OMax brings together many techniques in artificial intelligence, with an emphasis on machine listening (to translate audio information to symbolic information), and machine learning (to learn musical styles from human performers). Players can improvise alongside OMax in real time, with OMax generating music in a style learned from the player. Assayag describes the various skills that are required to produce an effective creative symbolic interaction system such as OMax, and outlines future directions for further improvement of such systems.

Boden's chapter is based on a previously published book chapter (Boden, 2004). The chapter describes the author's influential framework of creativity, and discusses its implications for computational creativity. Boden outlines three main types of creativity: combinatorial, exploratory, and transformational. Combinatorial creativity comes from the unfamiliar combination of familiar ideas; exploratory creativity refers to the exploration of new regions within a pre-established style of thought or conceptual space; transformational creativity alludes to a redefinition of the conceptual space. Boden describes how previous researchers have attempted to operationalize these different aspects of creativity in areas such as pun generation and pastiche music generation.

Andreatta's chapter reviews previous research on rhythmic tiling canons. Rhythmic canons are polyphonic structures where the same rhythm is played by multiple voices at different temporal displacements. Rhythmic tiling canons are rhythmic canons where only one voice sounds at any given moment. Andreatta describes how a well-known theorem of group theory can be used to construct rhythmic tiling canons where both the imitated rhythm and the timing of the voice entries correspond to regular patterns. The Vuza canon is then introduced, a class of rhythmic tiling canon where there is no periodicity in the imitated rhythm. The chapter concludes with a survey of compositional applications of rhythmic tiling canons.

\section{SHAPING PERFORMANCE}

Shaping Performance comprises three chapters. The first chapter, by Neta Spiro, Nicholas Gold, and John Rink, reproduces work from several earlier papers (Spiro, Gold, \& Rink, 2007a, 2007b). The chapter introduces the concept of performance motives, "expressive patterns in performative parameters such as timing, dynamics, articulation and timbre that maintain their identity upon identical or varied repetition" (p. 110). The authors present an algorithm for identifying performance motives in timing based on a knowledge representation scheme called formal concept analysis (FCA). FCA describes relationships between objects in terms of their distinct and shared attributes. In the authors' implementation, an object is a group of consecutive quaver lengths that occurs in a musical performance, and the attributes of an object are all other equally sized groups of quaver lengths that occur in the performance, within a given similarity threshold of the original group. The authors apply FCA primarily as a clustering algorithm, to find clusters of similarly timed quavers, which they identify as performance motives. This is an interesting idea, though it is unclear why the authors do not simply use a traditional clustering approach, such as hierarchical clustering or $k$ means. The authors identify a range of different types of performance motives in a series of performances of Chopin's Etude Op. 10, No. 3, and interpret some of these motives analytically.

The second chapter is contributed by Bernard Lanksey and Stephen Emmerson. It concerns mathematical metaphors for musical performance, and is centered on the authors' performance of a twopiano arrangement of Bach's Goldberg Variations at the Mathemusical Conversations workshop. The chapter begins with some observations about numerical relationships embedded in the piece, such as the role of the number 32 (the theme is 32 bars long, and the piece comprises 32 movements), and the fact that each third piece is a canon, with the intervals between the canonic voices increasing by one scale degree each canon. The authors then discuss how mathematics might play a role in a performance of this piece. An unusual connection is made to Einstein's general theory of relativity, based on the observation that the Goldberg Variations can be divided into 10 groups of 3 movements, and that Einstein's theory is based on 10 field equations. This prompts several additional analogies from the authors, such as Einstein's reconsideration of 
the axioms of Euclidean geometry being analogous to challenging the conception of the musical score as inviolate text. The authors illustrate their analogies with several musical examples.

The final chapter is contributed by Christopher Raphael, and describes the Informatics Philharmonic, an automatic musical accompaniment system that provides orchestral accompaniment to live performers (Raphael, 2010, 2011). The system uses hidden Markov models to follow the soloist's location in the score, a Bayesian belief network to predict upcoming events, and a phase vocoder to speed up or slow down the orchestral accompaniment. Raphael describes how the Informatics Philharmonic has been successfully used for several years at Indiana University's Summer String Academy, providing young musicians with the unique chance to rehearse and perform concerto movements without the necessity of booking a full symphony orchestra.

\section{EDUCATING THE MATHEMUSICAL}

Educating the Mathemusical comprises contributions from Jian Yang, Jeanne Bamberger, and Don McLean. Yang's chapter describes several mathematical and computational tools that he has used for the purposes of music education, including an interactive model of a plucked string, a 3D spectral model for visualizing the timbre of singing voices, a pitch-time model for visualizing a singer's intonation and vibrato, and tempotime/dynamics-time models for analyzing and comparing orchestral performances. Yang laments the fact that these tools have not proved as popular as he originally expected, and suggests that this might stem from a general antipathy of music students and teachers towards mathematical and empirical disciplines.

Bamberger's chapter is based on an earlier paper (Bamberger \& DiSessa, 2003), and concerns Impromptu, an interactive computer-music program. Impromptu was designed as part of a curriculum for teaching college-level music fundamentals courses. The main goal of this software was to help students develop and better understand their musical intuitions. One way of addressing this goal was through providing multiple complementary visualizations of musical structure. For example, a rhythmic pattern might be represented as a) numeric values corresponding to the length of each note in basic time units; b) a series of horizontally spaced vertical lines, with horizontal spacing proportional to inter-onset interval; c) a horizontal series of bars where the length of each bar is proportional to note length. Bamberger reports several occasions where she observed sixth-grade children using Impromptu spontaneously identifying rhythmic manifestations of mathematical phenomena such as equivalent ratios or lowest common multiples. She concludes that the multiple representations afforded in Impromptu provided a useful tool for students to benefit from implicit connections between mathematics and music.

McLean's chapter is an assortment of various ideas and observations about education, mathematics, and music. He discusses the common situation of students in higher education having to choose between pursuing music or another academic discipline, something he experienced himself with music and physics. In these cases, he usually recommends pursuing music, as musical study is particularly time-sensitive. McLean then introduces several interdisciplinary research centers that he has helped create, including the Center for Interdisciplinary Research in Music Media and Technology at McGill University, the Music and Health Research Collaboratory at the University of Toronto, and the Mind Brain Behaviour Hive at the University of Toronto. He describes some of the decisions that have led to success in these research centers. Next, McLean pays tribute to James Stewart, a research mathematician and musician based in Toronto, who often drew deep connections between mathematics and music. Lastly, McLean reflects on some overlaps between mathematics and music.

\section{GEOMETRIES}

Geometries comprises three chapters. The first, by Richard Cohn, reviews graph-theoretic and geometric models of pitch and tonality. Graph-theoretic models are mathematical structures that model pairwise relationships between objects (such as pitches and chords) in terms of networks of vertices connected by edges. Geometric models are mathematical models based on the notion of space and concepts such as distance, neighborhood, and connectivity. Cohn begins in Ancient Greece with Crantor's lambda, continues with Nicomachus's triangle, Torkesey's triangle, Euler's Tonnetz and its various adaptations, Douthett's Cube Dance, and finally concludes with Tymoczko's orbifold. The juxtaposition of so many models in such a short space could have produced chaotic results, but Cohn successfully draws a common historical thread throughout these models, describing how each model was a natural response to contemporaneous 
developments in musical practice. This chapter could serve well as a first introduction to this area of music modelling, perhaps as part of a university course in music theory or music psychology.

Dmitri Tymoczko's chapter concerns voice leading, the progression of individual voices in a polyphonic texture. Tymoczko begins by describing how voice leading can be formalized in terms of vectors in pitch-class space, an approach that the author has employed to great effect in previous work (e.g. Tymoczko, 2011). The remainder of the chapter explores several ways in which this vector formalization of voice leading can be useful. First, Tymoczko describes two corpus analyses investigating scale degree progressions in certain cadential patterns, such as $\mathrm{vii}^{\circ 6} \rightarrow \mathrm{I}$ and IV $\rightarrow \mathrm{I}$; in both cases, he finds that empirical data contradict common beliefs in pedagogical and music-theoretic practices. Next, Tymoczko introduces the concept of a canonic voice leading, a voice leading connecting two transpositionally related chords while acting as a cycle on their elements. Tymoczko demonstrates the use of canonic voice leadings in a madrigal by Luca Marenzio (“Ahi dispietata, morte!", 1585), and a chorale by J. S. Bach (number 115 in the Riemenschneider collection). Thirdly, Tymoczko discusses more broadly the utility of geometric approaches to understanding voice leading, highlighting the philosophy underlying his choice of models, and demonstrating how voice leading geometries can be generalized from chords to chord types (i.e. sets of chords that are equivalent under transposition). The chapter concludes with a summary of potential future directions in voice leading research.

The final chapter, contributed by Jean-Louis Giavitto and Antoine Spicher, reviews topological approaches to music theory. Topological models concern properties of spatial objects that are invariant to continuous deformations, such as stretching or bending. The authors begin by introducing the notion of cell spaces, assemblies of elementary objects called topological cells that are "glued" together. Several examples are then given of how cell spaces can be constructed to reflect aspects of music, either of general musictheoretic constructs (e.g. the 12 major chords), or of individual musical works (e.g. the temporal evolution of pitch, rhythm, and timbre throughout a given piece). At times the presentation is rather technical, relying heavily on the reader's prior mathematical knowledge. Nonetheless, readers without such a background can still appreciate some of the flavor of this approach to music modelling through the chapter.

\section{Discussion}

Mathemusical Conversations is an interdisciplinary book that provides a variety of perspectives on intersections between mathematics, computation, and music. Unusually for a book being reviewed in EMR, most chapters are not scientifically oriented: they do not concern falsifiable theories, and their conclusions are not based on objective analysis of empirical data [2]. This can provide a bit of a culture shock to the empirical musicologist used to hypothesis tests and $p$-values.

It is clear, however, that this relaxation of scientific principles permits a variety of academic contributions that would be impossible otherwise. For example, analogies play key roles in many chapters: Schoenfield compares composition to proving mathematical theorems, Chew compares musical structure to physical motion, and Lanskey and Emmerson compare Bach's Goldberg Variations to Einstein's general theory of relativity. Analogies are essentially untestable, and as a result are not scientific statements. Nonetheless, analogies can still be valuable, especially in music; they can provide listeners with new ways of "hearing" music, motivate visualizations that facilitate engagement and learning, and inspire composers to find new modes of creativity. Of course, analogy is not new to musicology, but "mathemusical" analogies are relatively uncommon, and it is refreshing to see so many presented here.

Similarly, removing the scientific obligation towards large sample sizes, objective measures, and inferential statistics allows several authors to make contributions based on their unique experiences in their fields of expertise. For example, Egozy writes of his experience of developing five musical games, describing a trade-off between engagement and expressivity in such games. In a scientific context, five data points would probably be insufficient to claim such a relationship, but relaxing scientific obligations allows us to make use of the extensive intuitions about creative game design that Egozy must have gained during his time at Harmonix. Likewise, Bamberger's chapter is based on personal observations made across six months working with a small group of children in an educational setting. This project originally had nothing to do with mathematics, and the observations described in Mathemusical Conversations came about by chance. These observations may not be scientifically rigorous - the sample size is small, and the observations are informal - but the author has a tremendous amount of experience in this area, and she spent a very long time with these children, so it makes sense to take her observations seriously. 
Many of these points where scientific perspectives are missing from Mathemusical Conversations might be inspiring to empirical musicologists for that very reason. Empirical studies could easily be conducted to test many of the hypotheses stated in these chapters, such as the assertions that engagement and creativity are inversely related in musical games (Ergozy), that music tuition can help students develop their mathematical intuitions (Bamberger), or that computational tools can provide a useful aid to music tuition (Yang).

The physical book is well-presented as a bound volume from World Scientific Publishing. It is unfortunately on the expensive side, especially for the UK readership. It benefits from attractive color figures which have generally printed well. The main deficiency in presentation comes from certain cases where figures are either too small or too low-resolution, sometimes rendering text unreadable as a result (e.g. pp. 51-53; p. 98; p. 117; pp. 121-124; p. 196). Nonetheless, there are still many cases of excellent figures (especially in Cohn's and Tymoczko's chapters).

In summary, I would recommend this book as a general introduction to various ways in which mathematics and computation might be combined with music. The lack of requirement for prior mathematical knowledge would make it well-suited for use as teaching material for university courses in music theory or music psychology, although its high price might make it impractical for use as a standard textbook. Empirical musicologists should find that Mathemusical Conversations provides a stimulating change in perspective from the usual scientific literature, as well as suggesting some interesting potential directions for future empirical work.

\section{Notes}

Peter M. C. Harrison Queen Mary University of London

[1] Given this suggestion, it is surprising that Schoenfield does not acknowledge the potential for probabilistic modelling (unarguably a subset of mathematics) as a composition method.

[2] The main exception is Tymoczko's chapter, which takes a strongly empiricist approach towards characterizing historic voice-leading practices.

\section{References}

Assayag, G. (2014). Music technology meets philosophy: From digital ethos to virtual ethos. In Proceedings of the Joint ICMC-SMC Conference (pp. 1-6).

Bamberger, J., \& DiSessa, A. (2003). Music as embodied mathematics: A study of a mutually informing affinity. International Journal of Computers for Mathematical Learning, 8(2), 123-160. https://doi.org/10.1023/B:IJCO.0000003872.84260.96

Boden, M. (2004). In a nutshell. In M. Boden, The Creative Mind: Myths and Mechanisms (pp. 1-10). London, England: Routledge.

Chew, E. (2000). Towards a mathematical model of tonality. (Unpublished doctoral dissertation). MIT, Cambridge, MA.

Chew, E. (2016). Playing with the edge: Tipping points and the role of tonality. Music Perception, 33(3), 344-366. https://doi.org/10.1525/mp.2016.33.3.344

Chew, E., François, A. R. J., Liu, J., \& Yang, A. (2005). ESP: A driving interface for expression synthesis. In Proceedings of the 2005 International Conference on New Interfaces for Musical Expression (NIME05) (pp. 224-227).

Raphael, C. (2010). Music Plus One and machine learning. In Proceedings of the 27th International Conference on Machine Learning (pp. 21-28). 
Raphael, C. (2011). The Informatics Philharmonic. Communications of the ACM, 54(3), 87-93. https://doi.org/10.1145/1897852.1897875

Spiro, N., Gold, N., \& Rink, J. (2007a). In search of motive: Identification of repeated patterns in performances and their structural contexts. In Proceedings of the International Conference on Music Communication Sciences (ICOMCS) (pp. 151-154).

Spiro, N., Gold, N., \& Rink, J. (2007b). Performance motives: Analysis and comparison of performance timing repetitions using pattern matching and Formal Concept Analysis. In Proceedings of the International Symposium on Performance Science.

Tymoczko, D. (2011). A Geometry of Music. New York, NY: Oxford University Press. 